\title{
Various approaches for prevention of Dental caries with emphasis on Probiotics: A review
}

\author{
Pravesh Bhardwaj, Srinath Krishnappa
}

\begin{abstract}
Aim: The aim of this comprehensive review is to present an update about the recent advances in the management of dental caries. Background: Despite the use of age old conventional physical and chemotherapeutic methods for caries management, dental caries still continues to be the most prevalent form of oral infectious disease. Thus, there is a need for additional approaches to deal with dental caries. Conclusion: Probiotics seem to be very effective in dealing with dental caries as revealed by the studies conducted recently in this discipline. More studies are needed to explore the use of probiotics appropriately in the field of dentistry.

Key words: dental caries, probiotics, streptococcus mutans
\end{abstract}

\section{Introduction}

Dental caries is one of the most common preventable childhood diseases; people are susceptible to this ailment throughout their lifetime (1). Dental caries can be defined as the localized destruction of susceptible dental hard tissues by acidic by-products from bacterial fermentation of dietary carbohydrates $(2,3)$. Endogenous bacteria (mainly mutans streptococci (Streptococcus mutans and Streptococcus sobrinus) and Lactobacillus spp.\} in the biofilm (dental plaque) produce weak organic acids as metabolic by-products of fermentable carbohydrates. The acids would cause local $\mathrm{pH}$ to fall below a critical value resulting in demineralisation of the tooth tissue (3).

Current methods of caries management include traditional prevention (prophylaxis, fluorides), early surgical intervention/minimal intervention, lesion monitoring and recall, and finally, delayed intervention (4). The main aim of this review is to highlight the role of conventional methods and probiotics in the prevention of dental caries.

\section{Chemoprophylactic agents}

Chemoprophylactic agents that are used in dental caries prevention include classical antibiotics such as penicillin and vancomycin; cationic agents such as chlorhexidine and cetylpyridinium chloride; plant derived compounds such as sanguinaria extract; anionic agents such as sodium dodecyl sulphate (SDS); and non-ionic agents such as triclosan (3).

Chlorhexidine is one of the most tested compounds and its anti-plaque properties are wellknown. In a supragingival biofilm model, chlorhexidine was shown to inhibit bacterial growth and biofilm formation. Because chlorhexidine is positively charged, it binds to various surfaces including enamel pellicle, hydroxyapatite and mucous membranes. A major part of the effectiveness of chlorhexidine is due to this substantivity (3).

Triclosan is the most commonly used and most potent example of the chlorinated diphenyl ether class of antibacterial compounds. Several large clinical trials have shown that toothpastes containing triclosan and zinc citrate significantly reduced plaque and gingival scores (3).

\section{Limitations of chemoprophylactic agents in caries prevention}

Chlorhexidine- Retention of chlorhexidine on tooth surface leads to an undesirable side-effect which is tooth staining and calculus formation, hypoguesia $(3,5)$.

Triclosan- Poor water solubility ( $11 \mu \mathrm{g} / \mathrm{ml}$, which is lower than its minimum inhibitory concentration (MIC) of $20 \mu \mathrm{g} / \mathrm{ml}$ ) and low retention (half-life for clearance is only $20 \mathrm{~min}$ ) in the oral cavity have limited its effectiveness and application for prevention and treatment of dental caries (3).

Chemotherapy- One of the most common problems in dental caries chemotherapy is the inability to maintain the minimum inhibitory concentration (MIC) of the drug in oral cavity (3). 
Antibiotics- The main concern regarding the use of antibiotics today is the progressive increase and proliferation of antibiotic-resistant organisms which significantly reduces the efficacy of conventional antibiotics (3).

\section{Antimicrobial peptides}

Recently, antimicrobial peptides (AMPs) have come to the forefront as potential antibiotic surrogates due to their robust killing activity against a wide spectrum of bacterial species, including drug-resistant strains. AMPs are genetically common molecules of innate immunity that have been discovered in single-cell and multicellular forms of life. These peptides may vary dramatically in peptide sequence and posttranslational modification (linear, circular, etc.), but the majority of them exhibit similar physical hallmarks including amphipathic mixtures of $\alpha$-helical and $\beta$-sheet structures and an overall cationic charge. Their mode of action often involves binding to the negatively charged moieties, e.g. lipopolysaccharide (LPS), on the microbial membrane. Once bound to the microbial surface, the peptides are predicted to lead to membrane disruption by insertion, but may also translocate into the microbe and kill by intracellular mechanisms. Due to their attraction to negatively charged structural molecules on the bacterial membrane, development of resistance to these peptides is rare (3).

\section{Limitations of Antimicrobial peptides in caries prevention}

Their application has been blocked by several issues including difficulty and expense of manufacturing, and short half-lives due to proteolytic degradation (3).

\section{Caries Vaccines}

Another line of defense in human body that can be utilized against $S$. mutans colonization is the specific antibody production from adaptive immunity. Immune defense in dental caries is mediated mainly by secretory $\operatorname{IgA}(\operatorname{sg} \mathrm{A})$ antibodies present in saliva and generated by the mucosal immune system. Mucosal immunization with $S$. mutans antigens at inductive sites, including gut-associated lymphoid tissue (GALT) and nasopharynx-associated lymphoid tissue (NALT), results in the migration of antigen-specific IgA-producing B cells to effector organs, such as the salivary glands. This is followed by the differentiation and maturation of these B cells and the secretion of IgA in the lamina propria, where it crosses the effector tissue ducts into the saliva. Results showed that vaccine or monoclonal antibody can effectively prevent $S$. mutans colonization. However, more clinical data regarding to caries experience are required before they can be proved effective against dental caries (3).

\section{Limitations of Vaccines in caries prevention}

Unwanted antibody response and allergy reactions, associated with non-human source monoclonal antibodies (3).

\section{Sugar Substitutes}

The formation of dental caries and the demineralization of tooth enamel are not caused by pathogenic bacteria itself, but most directly caused by the acids produced by acidogenic bacteria. Dietary sugars such as sucrose glucose and lactose can be fermented by microorganisms such as $S$. mutans and S. sobrinus to produce lactic acid which is most important and the strongest acid produced in large quantities that involved in the etiology of dental caries. Sugar substitutes that mainly used to prevent dental caries are xylitol and sorbitol. Xylitol cannot be utilized and fermented by microorganisms in oral cavity. Both in vitro and in vivo studies showed that the plaque $\mathrm{pH}$ is not affected by the intake of xylitol, and it was found that xylitol has a bacteriostatic effect on $S$. mutans by creating a futile cycle that consumes cellular ATP. Numerous clinical studies have shown that consumption of xylitol can significantly reduce saliva and plaque $S$. mutans levels. The major difference between sorbitol and xylitol is that, sorbitol can be fermented by oral microorganisms but the rate of acid production is significantly slower than that of other dietary sugars such as sucrose, glucose and fructose (3).

\section{Limitations of sugar substitutes in caries prevention}

The antibacterial properties of sugar substitutes are rather weak when compare to other therapeutic antimicrobial agent, therefore, prolonged exposure of these sugar substitutes in the oral cavity on a daily bases is required for them to be effective. Traditional delivering vehicles such as chewing-gums, hard candies and mints can only provide contact of the sugar substitutes with tooth surface for a few minute or even seconds. Therefore, novel delivery vehicles are still needed for the effective delivery of sugar substitutes before they can be considered as therapeutically effective (3).

\section{Casein Phosphopeptides}


Dairy products such as milk, milk concentrates and cheese are recognized as non-cariogenic or cariostatic in several laboratory studies due to the presence of milk phosphoprotein, casein. The casein phosphopeptides (CPP) are derived from casein by tryptic digestion. In 1987, Reynolds found that CPPs were incorporated into the intra-oral appliance plaque and were associated with a substantial increase in the plaque's content of calcium and phosphate. All CPPs contain the sequence motif -Pse-Pse-Pse-Glu-Glu-, where Pse is a phosphoseryl residue. Through these multiple phosphoseryl residues, CPPs have a marked ability to stabilize calcium phosphate ions in solution and to form a amorphous calcium phosphate (ACP) complex, referred to as CPP-ACP. It is proposed that the CPP bind to the forming alkaline ACP nanoclusters, producing a metastable solution thereby preventing ACP growth to the critical size required for nucleation and precipitation. CPP-ACP binds strongly to hydroxyapatite and can diffuse and retained in dental plaque, therefore is able to buffer acid and substantially raise the level of calcium phosphate in plaque or close proximity to the tooth surface, and thus inhibits enamel demineralization and enhances remineralization (3).

\section{Fluoride}

Fluoride therapy has been the main caries preventive strategy since the introduction of water fluoridation schemes several decades ago. ${ }^{3}$ Fluoride salts can be commonly found in drinking water, toothpastes and mouse rinses. However, it has been pointed out that, even with such a high availability, an increase in caries-free population reached a plateau in 1990s, and there were still at least $60 \%$ of teenagers around that time, and most likely still today, had observable dental decay. Thus the anti-caries effect of traditional fluoride therapy is still limited (3).

\section{Restorative methods}

Longevity of restorations is very low in the primary dentition (6). Generally, the earlier the age at restoration, the lower the longevity (7).

Table 1

\begin{tabular}{|c|c|c|c|c|c|c|}
\hline Author & year & Study period & $\begin{array}{l}\text { Number of } \\
\text { subjects }\end{array}$ & Restorations & Attrition & findings \\
\hline $\begin{array}{l}\text { Donly et al } \\
\text { (8) }\end{array}$ & 1999 & 3 years & 40 (6-9 years) & $\begin{array}{l}40 \text { amalgam } \\
40 \text { RMGI } \\
\text { Trad. Class-2 }\end{array}$ & $52.5 \%$ & $\begin{array}{l}\text { Am- 29.4\% failures, } \\
\text { RMGI- } 26.7 \% \text { failures (less } \\
\text { proximal demineralization) }\end{array}$ \\
\hline $\begin{array}{l}\text { Espelid et } \\
\text { al (9) }\end{array}$ & 1999 & 3 years & 43 (5-11 years) & $\begin{array}{l}49 \text { GI } \\
49 \text { RMGI } \\
\text { Proximal box only }\end{array}$ & $46 \%$ & $\begin{array}{l}\text { RMGI- } 2 \% \text { failures, } \\
\text { GI- } 26.5 \% \text { failures (defects and } \\
\text { caries) }\end{array}$ \\
\hline $\begin{array}{l}\text { Marks et al } \\
(10)\end{array}$ & 2000 & 1 year & $\begin{array}{l}43 \quad \text { (av. } 6.6 \\
\text { years) }\end{array}$ & $\begin{array}{l}53 \mathrm{GI} \\
52-\text { Compomer, } \\
\text { Box- only }\end{array}$ & $36.2 \%$ & $\begin{array}{l}\text { GI- } 8.3 \% \text { failures, } \\
\text { Compomer- } 9.7 \% \text { failures } \\
\text { (both recurrent caries and } \\
\text { fracture) }\end{array}$ \\
\hline
\end{tabular}

Probiotics

Probiotics are described as live micro-organisms which when administered in adequate numbers confer a health benefit on the host (FAO/WHO 2001). The term probiotic, meaning "for life," is derived from the Greek language. It was first used by Lilly and Stillwell in 1965 to describe "substances secreted by one microorganism which stimulates the growth of another" and thus was contrasted with the term antibiotic. The most commonly used strains belong to the genera Lactobacillus and Bifidobacterium, genera that are commonly found in the oral cavity, including caries lesions (11).

Mechanism of action in the oral cavity (11).

\section{$>$ Direct interactions in dental plaque}

- Involvement in binding of oral micro-organisms to proteins (biofilm formation).

- Action on plaque formation and on its complex ecosystem by competing and intefereing with bacteria-to-bacteria attachments.

-Involvement in metabolism of substrates (competing with oral micro-organisms for substrates available).

- Production of chemicals that inhibit oral bacteria (antimicrobial substances).

\section{$>$ Indirect probiotic actions in the oral cavity}

- Modulating systemic immune function.

- Effect on local immunity.

- Regulation of mucosal permeability. 
- Selection pressure on developing oral microflora towards colonization by less pathogenic species.

\section{New approaches to achieve probiotic effects}

Targeted antimicrobial therapy via a novel STAMP technology - Currently available antimicrobials exhibit broad spectrum killing properties Indiscriminate killing of all microbes by these conventional antimicrobials disrupts the ecological balance of the indigenous microbiota with unknown clinical consequences. These investigators formulated a new class of antimicrobials called Specifically Targeted Anti-Microbial Peptides (STAMPs). A "STAMP" is a fusion peptide with two moieties: a killing moiety made of a nonspecific antimicrobial peptide and a targeting moiety containing a species-specific binding peptide. The targeting moiety provides specific binding to a selected pathogen and facilitates the targeted delivery of an attached antimicrobial peptide (3).

Interference with signaling mechanisms - Several pathogenic properties of $S$. mutans are regulated by quorum sensing mechanism involving Competence Stimulating Peptide (CSP) as the signaling molecule Addition of a high concentration of CSP can interfere with signaling events of $S$. mutans and induce the death of the bacterium, thus exhibiting a potential beneficial effect against dental caries (3).

\section{Role of probiotics in prevention of dental caries}

A number of researchers are developing "probiotic" methods to treat the caries causing infection. "Probiotic", as used here, means that mechanisms are employed to selectively remove only the (odonto) pathogen while leaving the remainder of the oral ecosystem intact (12). One of the replacement therapy options entails the application of a genetically engineered "effector strain" of $S$. mutans that will replace the cariogenic or "wild strain" to prevent or arrest caries and to promote optimal remineralization of tooth surfaces that have been demineralised but that have not become cavitated. S. mutans strain BCS3-L1 was a genetically modified effector strain designed for use in replacement therapy to prevent dental caries. Recombinant DNA technology was used to delete the gene encoding lactate dehydrogenase in BCS3-L1 making it unable to produce lactic acid. This effector strain was also designed to produce elevated amounts of a novel peptide antibiotic called mutacin 1140 that gives it a strong selective advantage over most other strains of $S$. mutans (3). In another study Suzuki and kumuda et al showed that E. faecium WB2000 and JCM5804 and E. faecalis JCM5803 inhibited the biofilm formation by oral viridans group and mutans group streptococci but did not inhibit biofilm formation by $S$. oralis in the viridans group $(13,14)$. Suzuki and Nicholas et al found that $S$. salivarius M18 (formerly strain Mia) was a megaplasmid carrying oral probiotic exhibiting broad-spectrum inhibitory activity against several streptococcal pathogens, notably the caries-causing Streptococcus mutans (13, 15). Caglar et al. also showed that administration of probiotic bacterium Lactobacillus reuteri ATCC 55739 or Bifidobacterium DN-173010 induced significant reduction of cariogenic $S$. mutans in saliva (16). Petersson et al found that daily intake of milk supplemented with fluoride and/or probiotic bacteria might reverse soft and leathery primary root caries lesions (PRCL) in older adults (17). Singh et al found that probiotic ice-cream containing Bifidobacterium lactis Bb-12 ATCC27536 and Lactobacillus acidophilus La-5 could reduce the levels of certain caries-associated micro-organisms in saliva (18).

Keller et al had shown that the lactobacilli displayed co-aggregation activity and inhibited growth of clinical mutans streptococci. The growth inhibition was strain-specific and dependent on $\mathrm{pH}$ and cell concentration (19). Tahmourespour et al conducted a study to investigate the ability of biofilm formation among mutans and non mutans oral streptococci and to determine the effect of Lactobacillus acidophilus DSM 20079 as a probiotic strain on the adhesion of selected streptococcal strains on the surfaces, it was observed that the Lactobacillus acidophilus had more effect on adherence of mutans streptococci than non mutans streptococci with significant difference $(p<0.05)$. Adhesion reduction is likely due to bacterial interactions and colonization of adhesion sites with probiotic strain before the presence of streptococci. Authors suggested that adhesion reduction might be an effective way of decreasing cariogenic potential of oral streptococci (20). Lee do K and tanzer et al had shown that Bifidobacterium adolescentis SPM1005, Bifidobacterium longum SPM1207 and Lactobacillus paracasei DSMZ16671 had a strong growth-inhibiting effect against $S$. mutans $(21,22)$. Hillman et al found that the daily application of JH145, a naturally occurring LDH-deficient variant of $S$. rattus, could compete with $S$. mutans for its habitat on the tooth surface. So, S. rattus JH145 had potential as a probiotic for use in the prevention of dental caries (23). Glavina et al conducted a study to establish effect of 14 day consumption of commercially available yoghurt containing Lactobacillus rhamnosus ATCC53103 - LGG (Bioaktiv LGG, Dukat, Croatia) on Streptococcus mutans and Lactobacillus spp. salivary counts in children, it was found that daily consumption of yoghurt containing LGG have an inhibitory effect on oral pathogenic bacteria and may be beneficial in caries prevention (24). Lactic acid bacteria like Lactococcus lactis 
competitively inhibited S. mutans growth under nutritional deficiency, and the metabolites of S. mutans, including several exogenous molecular signals, enhanced the expression of genes related to nisin synthesis, nisA, nisB and nisI. Additionally, L. lactis effectively colonised the surface of tooth enamel, which showed substantially less decay with L. lactis adhesion compared to S. mutans adhesion $(25,26)$. Twetman et al carried out a study to assess the effectivity of probiotics in caries reduction in children and reported a significant caries reduction in 3 to 4 year-old children after 7 months of daily consumption of probiotic milk (27).

\section{Future Prospects}

In field of oral immunology, probiotics are being used as passive local immunization vehicles against dental caries. Recently, by means of systemic immunization with a multivalent vaccine, L. rhamnosus GG was chosen as the vehicle to harbor IgG because of its widely known health benefits in humans and animals. High titers of antibodies against human cariogenic bacteria, S. mutans and S. sobrinus, were produced in bovine colostrum by a vehicle of fermented milk. It was found that early mucosal colonization with $E$. coli bacteria stimulates the mucosal immune system to produce specific antibodies as well as non-specific secretory immunoglobulins. Regarding both studies, probiotics seems to improve oral immune response. In the present day technology has improved drastically. Very soon, people will be able to go into space to live on the planets like the moon. NASA of USA is carrying out research to develop probiotic products which enable humans live in space (11).

\section{Safety concerns}

Probiotics like Lactobacilli, bifidobacteria, and lactococci have generally been regarded as safe. There are other probiotic organisms, such as Enterococcus, Bacillus, and other spore-forming bacteria, as well as streptococci, that are not generally regarded as safe but have been

used as probiotics, their use is associated with increased risk of Bacteremia and endocarditis development (28). Till date only few studies were reported in relation to safety of probiotics in the field of dentistry. Mackay et al (29) reported the development of L. rhamnosus endocarditis (strain not specified) after a dental extraction in a 67 year old man with mitral regurgitation who was taking probiotic capsules daily. Although probiotics have an excellent overall safety record, they should be used with caution in certain patient groups - particularly neonates born prematurely or with immune deficiency (30).

\section{Why this paper is important to paediatric dentists}

In this review, we discussed the various approaches to prevent dental caries. It is essential for the paediatric dentists to know about the all the preventive measures against dental caries as they are the one who deal with the children and encounters dental caries very frequently. This review adds various preventive measures and their advantages-disadvantages to the knowledge of paediatric dentists. Out of all these approaches, probiotics seems to be very promising. 'An apple a day keeps doctor away' the famous quote now in recent years will definitely modify into 'Probiotics per day keeps doctor away'.

\section{Conclusion}

The research regarding the benefits of recent advances for the management of dental caries has been undertaken in the recent years. Out of all the above mentioned treatment modalities, probiotics have got immense potential in dealing with dental caries as revealed by the studies conducted recently in this discipline. More studies are needed to explore the use of probiotics appropriately in the field of dentistry.

\section{References}

[1]. Selwitz RH, Ismail AI, Pitts NB. Dental caries. Lancet 2007; 369: 51-9.

[2]. Marsh, P.; Martin, MV. Oral Microbiology. 1999; 4th edn. Wright; Oxford.

[3]. Chen F and Wang D. Novel technologies for the prevention and treatment of dental caries: a patent survey. Expert Opin Ther Pat. 2010; 20: 681-694.

[4]. Gupta S, Marwah N. 'Use a Thorn to Draw Thorn' Replacement Therapy for Prevention of Dental Caries. International Journal of Clinical Pediatric Dentistry, 2010; 3: 125-137.

[5]. Yates R, Jenkins S, Newcombe R, et al. A 6-month home usage trial of a 1\% chlorhexidine toothpaste (1). Effects on plaque, gingivitis, calculus and toothstaining. J Clin Periodontol 1993; 20: 130-8.

[6]. Wendt L, Goran K, Birkhed D. Replacements of restorations in the primary and young permanent dentition. Swed Dent J 1998; 22: $149-55$.

[7]. Wong FSL, Day SJ. An investigation of factors influencing the longevity of restorations in primary molars. J Int Assoc Dent Child 1990; 20: 11-6.

[8]. Donly KJ, Segura A, Kanellis M, Erickson RL. Clinical performance and caries inhibition of resin-modified glass ionomer cement and amalgam restorations. J Am Dent Assoc 1999; 130: 1459-66.

[9]. Espelid I, et al. Clinical behaviour of glass ionomer restorations in primary teeth. J Dent 1999; 27: 437-42.

[10]. Marks LAM, et al. Ketac Molar versus Dyract class II restorations in primary molars: twelve-month clinical results. J Dent Child 2000; 67: 37-40

[11]. Reddy JJ, Sampathkumar N, Aradhya S. Probiotics in dentistry: review of the current status. Rev Clín Pesq Odontol 2010; 6: 261-7. 
[12]. Anderson MH, Shi WA. Probiotic approach to caries management. Pediatr Dent 2006; 28: 151-3.

[13]. Suzuki N, Yoneda M, Hatano Y, Iwamoto T, Masuo Y, and Hirofuji T. Enterococcus faecium WB2000 Inhibits Biofilm Formation by Oral Cariogenic Streptococci. Int J dent. 2011; 2011: 1-5.

[14]. Kumada M, Motegi M, Nakao R et al. "Inhibiting effects of Enterococcus faecium non-biofilm strain on Streptococcus mutans biofilm formation". J Microbiol Immunol Infect, 2009; 42: 188-196.

[15]. Heng NCK et al. Genome Sequence of the Bacteriocin-Producing Oral Probiotic Streptococcus salivarius Strain M18. J Bact 2011; 193: 6402-6403.

[16]. Caglar E, Kargul B, Tanboga I. Bacteriotherapy and probiotics' role on oral health. Oral Dis. 2005; 11: 131-7.

[17]. Petersson LG, Magnusson K, Hakestam U, Baigi A, Twetman S. Reversal of primary oot caries lesions after daily intake of milk supplemented with fluoride and probiotic lactobacilli in older adults. Acta Odontol Scand. 2011; 69: 321-7.

[18]. Singh RP, Damle SG, Chawla A. Salivary mutans streptococci and lactobacilli modulations in young children on consumption of probiotic ice-cream containing Bifidobacterium lactis Bb12 and Lactobacillus acidophilus La5. Acta Odontol Scand. 2011; 69: 38994.

[19]. Keller MK, Hasslöf P, Stecksén-Blicks C, Twetman S. Co-aggregation and growth inhibition of probiotic lactobacilli and clinical isolates of mutans streptococci: an in vitro study. Acta Odontol Scand. 2011; 69: 263-8

[20]. Tahmourespour A, Kermanshahi RK. The effect of a probiotic strain (Lactobacillus acidophilus) on the plaque formation of oral Streptococci. Bosn J Basic Med Sci. 2011; 11: 37-40.

[21]. Lee do K et al. Antimicrobial activity of Bifidobacterium spp. isolated from healthy adult Koreans against cariogenic microflora. Arch Oral Biol. 2011 56: 1047-54.

[22]. Tanzer JM. Caries inhibition by and safety of Lactobacillus paracasei DSMZ16671. J Dent Res. 2010; 89: 921-6.

[23]. Hillman JD, McDonell E, Cramm T, Hillman CH, Zahradnik RT. A spontaneous lactate dehydrogenase deficient mutant of Streptococcus rattus for use as a probiotic in the prevention of dental caries. J Appl Microbiol. 2009; 107: 1551-8.

[24]. Glavina D, Gorseta K, Skrinjarić I, Vranić DN, Mehulić K, Kozul K. Effect of LGG yoghurt on Streptococcus mutans and Lactobacillus spp. salivary counts in children. Coll Antropol. 2012; 36: 129-32.

[25]. Tong Z, Zhou L, Li J, Kuang R, Lin Y, Ni L. An in vitro investigation of Lactococcus lactis antagonizing cariogenic bacterium Streptococcus mutans. Arch Oral Biol. 2012; 57: 376-82.

[26]. Bosch $\mathrm{M}$ et al. Isolation and characterization of probiotic strains for improving oral health. Arch Oral Biol. 2012; 57: 539-49.

[27]. Twetman S, Stecksen-Blicks C. Probiotics and oral health effects in children. Int J Paediatr Dent 2008; 18: 3-10.

[28]. Snydman DR. The safety of probiotics. Clinical Infectious Diseases 2008; 46: 104-111.

[29]. Mackay AD, Taylor MB, Kibbler CC, Hamilton-Miller JM. Lactobacillusendocarditis caused by a probiotic organism. Clin Microbiol Infect 1999; 5: 290-2.

[30]. Robert JB, Roy MRB, and Mimi LKT. Probiotic use in clinical practice: what are the risks? Am J Clin Nutr 2006; 83: $1256-64$. 